Sädhanā Vol. 29, Part 4, August 2004, pp. 355-363. @ Printed in India

\title{
Propagation of Love waves in an elastic layer with void pores
}

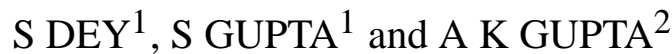 \\ ${ }^{1}$ Department of Applied Mathematics and ${ }^{2}$ Department of Applied Geophysics, \\ Indian School of Mines, Dhanbad 826 004, India \\ e-mail: d_sukhendu@ @otmail.com
}

MS received 18 June 2003; revised 3 March 2004

\begin{abstract}
The paper presents a study of propagation of Love waves in a poroelastic layer resting over a poro-elastic half-space. Pores contain nothing of mechanical or energetic significance. The study reveals that such a medium transmits two types of love waves. The first front depends upon the modulus of rigidity of the elastic matrix of the medium and is the same as the love wave in an elastic layer over an elastic half-space. The second front depends upon the change in volume fraction of the pores. As the first front is well-known, the second front has been investigated numerically for different values of void parameters. It is observed that the second front is many times faster than the shear wave in the void medium due to change in volume fraction of the pores and is significant.
\end{abstract}

Keywords. Love waves; elastic matrix; void pores; volume fraction of pores; equilibrated inertia; wave fronts.

\section{Introduction}

Linear and nonlinear theories of poro elastic material were introduced by Cowin and Nunziato around the year 1980 (Cowin \& Nunziato 1983, Nunziato \& Cowin 1979). Cowin (1985) also extended the theory to show that linear elastic materials with voids behave like viscoelastic materials. Scalia (1994) studied the propagation of shock waves in viscoelastic materials with voids. Chandrasekharaiah (1987) studied the effect of surface stress and voids on Rayleigh waves in elastic solids with voids. It was pointed out in his paper that there may be two longitudinal wave fronts in such a medium. Dey \& Gupta (1987) have studied the propagation of longitudinal and shear waves in void media and came to the conclusion that there may be two wave fronts for longitudinal waves. Dey et al (1993) discussed the propagation of torsional surface waves in an elastic medium with void pores. The influence of local irregularities on propagation of Love waves has been studied by Yu et al (1996). The propagation of Love waves in homogeneous and non-homogeneous elastic media has been studied by many authors. Some of these studies are available in Ewing et al (1957), Achenbach (1973), Pillant (1979) etc.

The present paper attempts to examine Love waves in elastic media containing voids. The mechanical constitutive equation satisfying the physical properties has been given by Cowin $\&$ Nunziato (1983). The velocity equation of Love waves in a poro-elastic layer over a poroelastic half-space has been obtained. It is shown that there is possibility of propagation of 


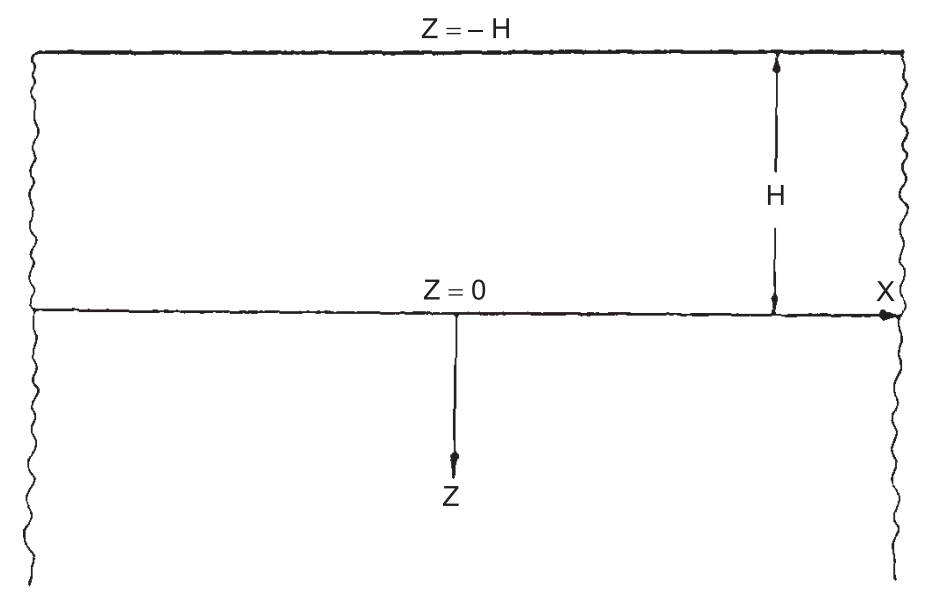

Figure 1. Geometry of the problem.

two wave fronts, one front associated with the elasticity of the medium and the other front to change in void volume.

\section{Summary of the theory}

The linear theory of elastic material with voids (Cowin \& Nunziato 1983) deals with small changes from a reference configuration of a porous body (see figure 1). In this configuration, the bulk density $\rho$, matrix density $\gamma$ and matrix volume fraction $v$ are related by

$$
\rho_{R}=\gamma_{R} v_{R},
$$

and the body is taken to be strain-free, although not necessarily stress-free. The independent kinematic variables in the linear theory are the displacement field $u_{i}(x, t)$ from the reference configuration and change in volume fraction from the reference volume fraction, $\phi(x, t)$ :

$$
\phi(x, t)=v(x, t)-v_{R}(x),
$$

where $x$ is the spatial position vector in Cartesian co-ordinates and $t$ is time. The infinitesimal strain tensor $E_{i j}(x, t)$ is determined from the displacement field $u_{i}$ according to

$$
E_{i j}=\frac{1}{2}\left(u_{i, j}+u_{j, i}\right)
$$

where the comma followed by a lowercase letter indicates partial derivative with respect to the indicated co-ordinate.

Assuming that the region occupied by a body is regular, the equations of motion governing a linear elastic continuum with void are the balance of linear momentum,

$$
\rho \frac{\partial^{2} u_{i}}{\partial t^{2}}=T_{i j, j}+b_{i}
$$

and the balance of equilibrated force,

$$
\rho \bar{k} \frac{\partial^{2} \phi}{\partial t^{2}}=h_{i, i}+g+\rho l .
$$


Here,

$T_{i j}$ is the symmetric stress tensor,

$b_{i}$ is the body force vector,

$h_{i}$ is the equilibrated stress vector,

$\bar{k}$ is the equilibrated inertia,

$g$ is the intrinsic equilibrated body force,

$l$ is the extrinsic equilibrated body force.

The constitutive equations for the stress tensor $T_{i j}$, the equilibrated stress vector $h_{i}$ and the intrinsic equilibrated body force $g$ to the strain $E_{i j}$, the change in volume fraction $\phi$, the time rate of change of the volume fraction $\phi$ and the gradient of the change in volume fraction $\phi, i$.

Thus,

$$
\begin{aligned}
T_{i j} & =C_{i j k m} E_{k m}+D_{i j k} \phi,_{k}+B_{i j} \phi, \\
h_{i} & =A_{i j} \phi_{, j}+D_{i j k} E_{j k}+f_{i} \phi, \\
g & =-\omega(\partial \phi / \partial t)-\xi \phi-B_{i j} E_{i j}-f_{i} \phi,{ }_{i},
\end{aligned}
$$

where $C_{i j k m}, B_{i j}, A_{i j}, f_{i}, \omega$ and $\xi$ are functions of $v_{R}$. If the material symmetry is of a type that possesses a centre of symmetry, then the tensor $D_{i j k}$ and $f_{i}$ are identically zero and the constitutive equations (6)-(8) are simplified. If, in addition, the material is isotropic in its dependence of $T_{i j}, h_{i}$ and $g$ on $E_{i j}, \phi,,_{i}$, then $C_{i j k m}, A_{i j}$ and $b_{i j}$ are given by

$$
\begin{aligned}
C_{i j k m} & =\lambda \delta_{i j} \delta_{k m}+\mu\left(\delta_{i k} \delta_{j m}+\delta_{i m} \delta_{j k}\right), \\
A_{i j} & =\alpha \delta_{i j}, \\
B_{i j} & =\beta \delta_{i j},
\end{aligned}
$$

where $\lambda, \mu$ are Lame's constants of elastic frame and $\alpha$, and $\beta$ are functions of $v_{R}$, and the constitutive equations (6)-(8) become

$$
\begin{aligned}
T_{i j} & =\delta_{i j} E_{k k}+2 \mu E_{i j}+\beta \quad \phi \delta_{i j}, \\
h_{i} & =\alpha \quad \phi,{ }_{i}, \\
g & =-\omega(\partial \phi / \partial t)-\xi \quad \phi-\beta E_{k k}, \\
\mu & \geqslant 0, \alpha \geqslant 0, \xi \geqslant 0, \bar{k} \geqslant 0, \bar{k} \xi \geqslant \beta^{2}, \omega \geqslant 0,
\end{aligned}
$$

where

$$
\bar{k}=\lambda+(2 / 3) \mu \text {. }
$$

The field equations governing the displacement field $u_{i}(x, t)$ and the volume fraction field $\phi(x, t)$ are obtained by substituting the constitutive relations (10)-(12) into the equations of motion (4) and (5) as

$$
\begin{aligned}
(\lambda+\mu) \nabla \nabla \cdot \bar{u}+\mu \nabla^{2} \bar{u}+\beta \nabla \phi & =\rho\left(\partial^{2} \bar{u} / \partial t^{2}\right), \\
\alpha \nabla^{2} \phi-\omega(\partial \phi / \partial t)-\xi \phi-\beta \nabla \cdot u & =\rho \bar{k}\left(\partial^{2} \phi / \partial t^{2}\right) .
\end{aligned}
$$


The boundary condition on $\phi$ is

$$
\bar{n} . \nabla \phi=0,
$$

where $\bar{n}$ is the unit vector normal to the external boundary and the boundary conditions on $\bar{u}$ are those of classical elasticity.

\section{Formulation}

Consider an elastic layer of thickness $H$ with void pores. The $z$-axis is taken vertically downward in the lower medium. The $x$-axis is chosen parallel to the layer in the direction of the wave propagation. Origin is chosen at the interface of the layer and the half-space.

The displacement components for Love waves are $u=0, w=0$ and $v=v(x, z, t)$. The equations of motion (14) and (15), which are not identically satisfied, under no body forces take the form,

$$
\begin{aligned}
\rho \frac{\partial^{2} v}{\partial t^{2}} & =\mu\left(\frac{\partial^{2} v}{\partial x^{2}}+\frac{\partial^{2} v}{\partial z^{2}}\right)+\beta\left(\frac{\partial \phi}{\partial x}+\frac{\partial \phi}{\partial z}\right), \\
\rho \bar{k} \frac{\partial^{2} \phi}{\partial t^{2}} & =\alpha\left(\frac{\partial^{2} \phi}{\partial x^{2}}+\frac{\partial^{2} \phi}{\partial z^{2}}\right)-\omega \frac{\partial \phi}{\partial t}-\xi \phi .
\end{aligned}
$$

\section{Solution}

For waves propagating in the positive direction of $x$-axis with velocity $c$ the solution of (17) and (18) may be taken as

$$
\begin{aligned}
v & =\bar{v}(z) e^{i k(x-c t),} \\
\phi & =\bar{\phi}(z) e^{i k(x-c t),}
\end{aligned}
$$

where $\bar{v}(z)$ and $\bar{\phi}(z)$ satisfy the equations,

$$
\bar{v}^{\prime \prime}(z)-N^{2} \bar{v}(z)+B\left[i k \bar{\phi}(z)+\bar{\phi}^{\prime}(z)\right]=0,
$$

with the values of $N, B$ as

$$
N=k\left(1-\left(c^{2} / A^{2}\right)\right)^{1 / 2}, \quad B=\beta / \mu, \text { and } A=(\mu / \rho)^{1 / 2}
$$

and

$$
\bar{\phi}^{\prime \prime}(z)-M^{2} \bar{\phi}(z)=0
$$

where

$$
M=\left[\left(\alpha k^{2}-\rho \bar{k} k^{2} c^{2}-i \omega k c t+\xi\right) / \alpha\right]^{1 / 2}
$$

$\alpha, \bar{k}, \xi$ being constants for a particular material. Ignoring the damping term $\omega$ which is very small for sinusoidal wave, the value of $M$ may be taken as

$$
M=k\left[1-\frac{c^{2}}{(\alpha / \rho \bar{k})}+\frac{1}{k^{2}(\alpha / \xi)}\right]^{1 / 2}
$$


The solution of (20) with $M$ as (21) may be taken as

$$
\bar{\phi}=R_{3} e^{M z}+R_{4} e^{-M z} \text {. }
$$

Using (22), the solution of (19) is

$$
\bar{v}=R_{1} e^{N z}+R_{2} e^{-N z}-\frac{B(i k+M)}{M^{2}-N^{2}} e^{M z} R_{3}-\frac{B(i k-M)}{M^{2}-N^{2}} e^{-M z} R_{4} .
$$

Hence the solution of (17) and (18) may be written as

$$
\begin{aligned}
v & =\left[R_{1} e^{N z}+R_{2} e^{-N z}-\frac{B(i k+M)}{M^{2}-N^{2}} e^{M z} R_{3}-\frac{B(i k-M)}{M^{2}-N^{2}} e^{-M z} R_{4}\right] e^{i k(x-c t)} \\
\phi & =\left[R_{3} e^{M z}+R_{4} e^{-M z}\right] e^{i k(x-c t)}
\end{aligned}
$$

\section{Solution for the upper layer}

Denoting the quantities for the upper layer by subscript 1 , the solution may be written as

$$
\begin{aligned}
v_{1}= & {\left[R_{1} \exp \left(N_{1} z\right)+R_{2} \exp \left(-N_{1} z\right)-\frac{B_{1}\left(i k+M_{1}\right) \exp \left(M_{1} z\right)}{M_{1}^{2}-N_{1}^{2}} R_{3}\right.} \\
& \left.-\frac{B_{1}\left(i k-M_{1}\right) \exp \left(-M_{1} z\right)}{M_{1}^{2}-N_{1}^{2}} R_{4}\right] \exp \{i k(x-c t)\}, \\
\phi_{1}= & {\left.\left[R_{3} \exp \left(M_{1} z\right)+R_{4} \exp \left(-M_{1} z\right)\right] \exp \{i k(x-c t)\}\right\}, }
\end{aligned}
$$

where

$$
\begin{aligned}
& N_{1}=k\left(1-\left(c^{2} / A_{1}^{2}\right)\right)^{1 / 2}, A_{1}=\left(\mu_{1} / \rho_{1}\right)^{1 / 2}, \\
& M_{1}=k\left[1-\frac{c^{2}}{\left(\alpha_{1} / \rho_{1} k_{1}\right)}+\frac{1}{k^{2}\left(\alpha_{1} / \xi_{1}\right)}\right]^{1 / 2}, B_{1}=\frac{\beta_{1}}{\mu_{1}} .
\end{aligned}
$$

\section{Solution for the half-space}

Denoting the quantities by the subscript 2 for the half-space and keeping in view that $\phi \rightarrow 0$ as $z \rightarrow \infty$, and $v \rightarrow 0$ as $z \rightarrow \infty$, the solution may be taken as

$$
\begin{aligned}
v_{2} & =\left[R_{5} \exp \left(-N_{2} z\right)-\frac{B_{2}\left(M_{2}-i k\right) \exp \left(-M_{2} z\right)}{M_{2}^{2}-N_{2}^{2}} R_{6}\right] \exp \{i k(x-c t)\}, \\
\phi_{2} & =R_{2} \exp \left(-M_{2} z\right) \exp \{i k(x-c t)\},
\end{aligned}
$$

where

$$
\begin{aligned}
& N_{2}=k\left(1-\left(c^{2} / A_{2}^{2}\right)\right)^{1 / 2}, A_{2}=\left(\mu_{2} / \rho_{2}\right)^{1 / 2}, \\
& M_{2}=k\left[1-\frac{c^{2}}{\left(\alpha_{2} / \rho_{2} k_{2}\right)}+\frac{1}{k^{2}\left(\alpha_{2} / \zeta_{2}\right)}\right]^{1 / 2}, B_{2}=\frac{\beta_{2}}{\mu_{2}} .
\end{aligned}
$$




\section{Boundary conditions}

Boundary conditions to be satisfied by the propagation are

$$
\left.\begin{array}{ll}
\text { (i) } & \left(\tau_{y z}\right)_{1}=\left(\tau_{y z}\right)_{2} \\
\text { (ii) } & (\bar{n} \cdot \nabla \phi)_{1}=(\bar{n} \cdot \nabla \phi)_{2} \\
\text { (iii) } & (\phi)_{1}=(\phi)_{2} \\
\text { (iv) } & (v)_{1}=(v)_{2}
\end{array}\right] \text { at } z=0 \text {, }
$$

and

$$
\left.\begin{array}{ll}
\text { (v) } & (\bar{n} \cdot \nabla \phi)_{1}=0 \\
\text { (vi) } & \left(\tau_{y z}\right)_{1}=0
\end{array}\right] \text { at } z=-H .
$$

Using (26) to (29), the above boundary conditions (30) and (31) give

$$
\begin{aligned}
& \mu_{1}\left[R_{1} N_{1}-R_{2} N_{1}+\frac{B_{1}\left(i k+M_{1}\right) M_{1}}{M_{1}^{2}-N_{1}^{2}} R_{3}+\frac{B_{1}\left(i k-M_{1}\right) M_{1}}{M_{1}^{2}-N_{1}^{2}} R_{4}\right] \\
& \quad+\mu_{2}\left[R_{5} N_{2}+\frac{B_{2}\left(M_{2}-i k\right) M_{2}}{M_{2}^{2}-N_{2}^{2}} R_{6}\right]=0 \\
& M_{1} R_{3}-M_{1} R_{4}+M_{2} R_{6}=0 \\
& R_{3}+R_{4}-R_{6}=0 \\
& R_{1}+R_{2}-\frac{B_{1}\left(i k+M_{1}\right)}{M_{1}^{2}-N_{1}^{2}} R_{3}-\frac{B_{1}\left(i k-M_{1}\right)}{M_{1}^{2}-N_{1}^{2}} R_{4}-R_{5}-\frac{B_{2}\left(M_{2}-i k\right)}{M_{2}^{2}-N_{2}^{2}} R_{6}=0 \\
& R_{3} \exp \left(-M_{1} H\right)-R_{4} \exp \left(M_{1} H\right)=0
\end{aligned}
$$

and

$$
R_{1} \exp \left(-N_{1} H\right)-R_{2} \exp \left(N_{1} H\right)=0 .
$$

For non-zero solution of $R_{1}, R_{2}, R_{3}, R_{4}, R_{5}$ and $R_{6}$ we have

$$
\left|\begin{array}{cccccc}
N_{1} & -N_{1} & -\frac{B_{1}\left(i k+M_{1}\right) M_{1}}{M_{1}^{2}-N_{1}^{2}} & \frac{B_{1}\left(i k-M_{1}\right) M_{1}}{M_{1}^{2}-N_{1}^{2}} & \frac{\mu_{2}}{\mu_{1}} N_{2} & \frac{\mu_{2} B_{2}\left(M_{2}-i k\right) M_{2}}{\mu_{1}\left(M_{2}^{2}-N_{2}^{2}\right)} \\
0 & 0 & M_{1} & -M_{1} & 0 & M_{2} \\
0 & 0 & 1 & 1 & 0 & -1 \\
1 & 1 & \frac{B_{1}\left(i k+M_{1}\right)}{M_{1}^{2}-N_{1}^{2}} & \frac{-B_{1}\left(i k-M_{1}\right)}{M_{1}^{2}-N_{1}^{2}} & -1 & \frac{-B_{2}\left(M_{2}-i k\right)}{\left(M_{2}^{2}-N_{2}^{2}\right)} \\
0 & 0 & e^{-M_{1} H} & e^{M_{1} H} & 0 & 0 \\
e^{-N_{1} H} & e^{N_{1} H} & 0 & 0 & 0 & 0
\end{array}\right|=0 .
$$

On evaluation, the determinant gets factorised into

$$
\begin{aligned}
& {\left[\mu_{2} N_{2} \exp \left(N_{1} H\right)-\left(\mu_{2} N_{2}-\mu_{1} N_{1}\right) \sinh \left(N_{1} H\right)\right]\left[-M_{1} H \sinh \left(M_{1} H\right)\right.} \\
& \left.\quad+M_{2}\left\{-\exp \left(M_{1} H\right)+\sinh \left(M_{1} H\right)\right\}\right]=0 .
\end{aligned}
$$


Hence, either

$$
\left(N_{2} \mu_{2}-N_{1} \mu_{1}\right) \sinh \left(N_{1} H\right)-\mu_{2} N_{2} \exp \left(N_{1} H\right)=0,
$$

or

$$
\left(M_{2}-M_{1}\right) \sinh \left(M_{1} H\right)-M_{2} \exp \left(M_{1} H\right)=0 .
$$

Equation (38) on simplification gives

$$
\tan \left[\left(\frac{c^{2}}{A_{1}^{2}}-1\right)^{1 / 2}\right] k H=\frac{\left(1-\left(c^{2} / A_{2}^{2}\right)\right)^{1 / 2}}{\left(\left(c^{2} / A_{1}^{2}\right)-1\right)^{1 / 2}} \frac{\mu_{2}}{\mu_{1}}
$$

and (39) takes the form

$$
\tan \left[\left(\frac{c^{2}}{c_{3}^{2}}-1-\frac{1}{(k m)_{1}^{2}}\right)\right]^{1 / 2} k H=\frac{\left[1-\left(c^{2} / c_{3}^{2}\right) S+\left(1 /(k m)_{2}^{2}\right)\right]^{1 / 2}}{\left[\left(c^{2} / c_{3}^{2}\right)-1-\left(1 /(k m)_{1}^{2}\right)\right]^{1 / 2}}
$$

where

$$
\begin{aligned}
(\mathrm{km})_{1}^{2}=k^{2}\left(\alpha_{1} / \xi_{1}\right),(\mathrm{km})_{2}^{2}=k^{2}\left(\alpha_{2} / \xi_{2}\right), & \\
c_{3}=\left(\alpha_{1} / \rho_{1} k_{1}\right)^{1 / 2}, & \text { velocity of shear wave due to change in void volume } \\
& \text { fraction in the layer, } \\
\bar{c}_{3}=\left(\alpha_{2} / \rho_{2} k_{2}\right)^{1 / 2}, & \text { velocity of shear wave due to change in void volume } \\
& \text { fraction in the half-space, }
\end{aligned}
$$

and

$$
S=c_{3}^{2} / \bar{c}_{3}^{2}
$$

The study shows that Love waves propagate in elastic media with void pores in two wave fronts. One wave front, given by (41), is associated with the parameters of the void pores which are involved with the change of void volume fraction and the equilibrated inertia. The other wave front given in (40) is the same as it would have been in elastic half-space without pores. Both the fronts are dispersive in nature. As a particular case, in the absence of void pores (41) takes the form of (40) which is the well-known dispersive equation for Love waves in an elastic layer over an elastic half space.

\section{Numerical calculation}

The numerical values of $c^{2} / c_{3}^{2}$ have been calculated from (41) for different values of $k H$ taking some sets of values of $(\mathrm{km})_{1},(\mathrm{~km})_{2}$ and $S$. The results are presented in figures 2 and 3 . The figures show that the velocity of Love wave front which depends on the change in volume of void pores is many times higher then the velocity of waves carrying a change in void fraction of pores at low values of $k H$. 


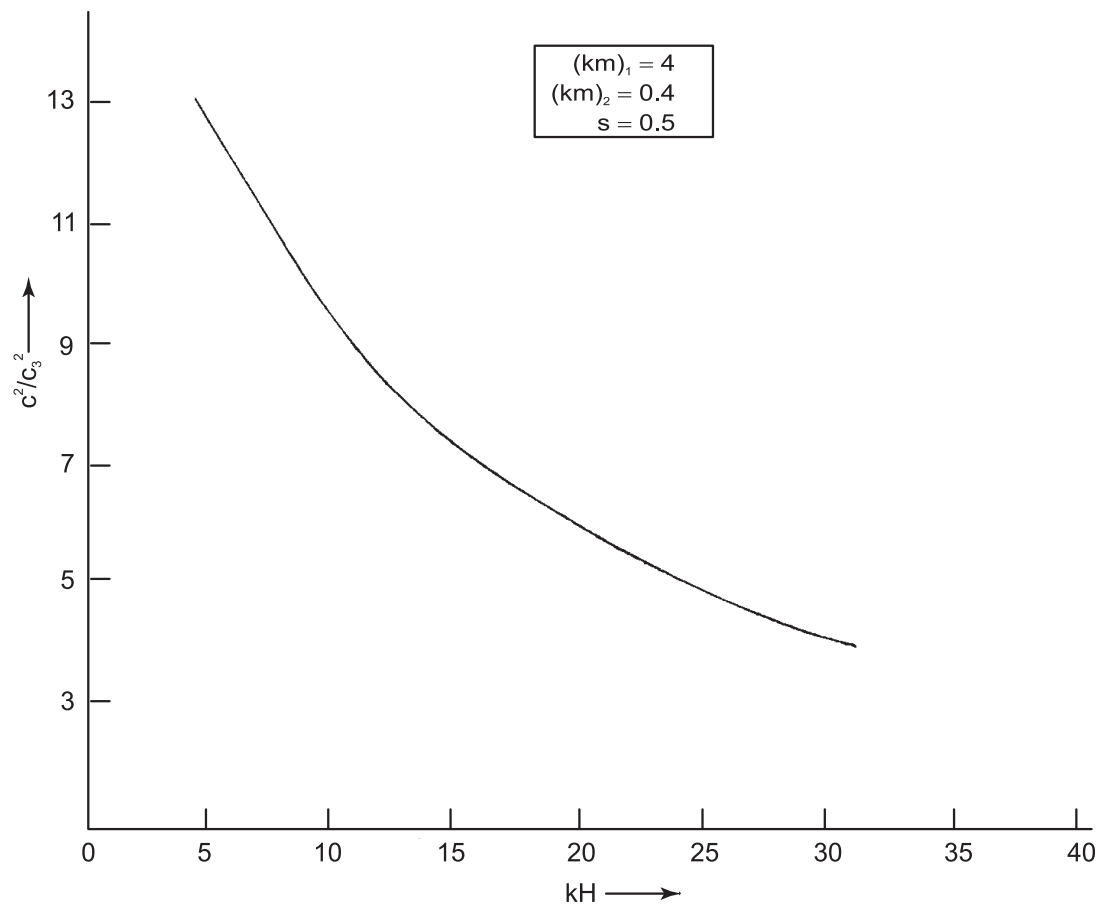

Figure 2. Love wave dispersion curve in an elastic medium with void pores for a set of parameters.

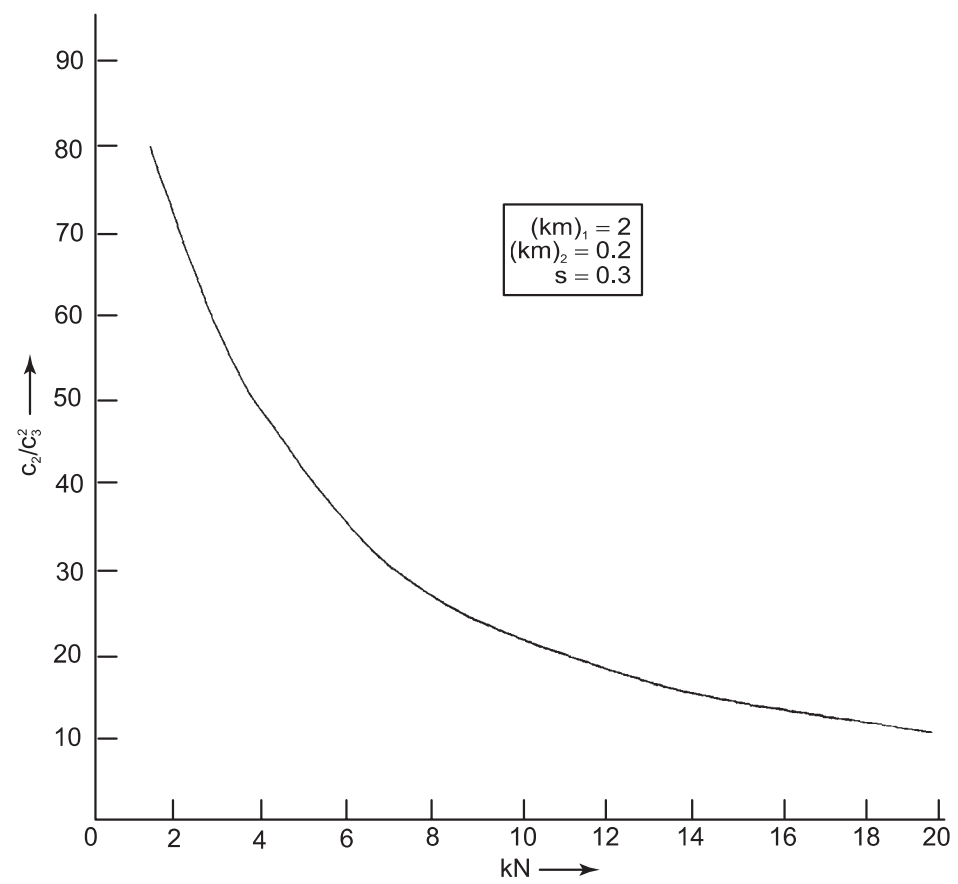

Figure 3. Love wave dispersion curve in an elastic medium with void pores for a second set of parameters. 


\section{Conclusion}

The study arrived at the following results:

(1) The two Love wave fronts may be available in the medium. The velocity of the first depends on the rigidity of the elastic medium and is the same as Love waves in elastic medium. The other depends on the change in void volume fraction of pores.

(2) The velocity of Love wave fronts of second type is much more than the velocity of shear waves due to the change in void volume fraction of pores and deserves consideration.

(3) The velocity depends on the ratio of void volume fraction of pores of the layer and halfspace and is increased if the ratio decreases. Since some regions of the layers of the earth are dry and porous without the filling of any significant materials, the possibility of existence of two Love wave fronts in such a layer attracts the attention of seismologists.

\section{References}

Achenbach J D 1973 Wave propagation in elastic solids (New York: North Holland)

Chandrasekharaiah D S 1987 Effects of surface stresses and voids on Rayleigh waves in elastic solid. Int. J. Eng. Sci. 25: 205-211

Cowin S C 1985 The viscoelastic behaviour of linear elastic materials with voids. J. Elasticity 15: 185-191

Cowin S C, Nunziato J W 1983 Linear elastic materials with voids. J. Elasticity 13: 125

Dey S, Gupta S 1987 Longitudinal and shear waves in an elastic medium with void pores. Proc. Indian Natl. Sci. Acad. A53: 554-563

Dey S, Gupta S, Gupta A K 1993 Torsional surface waves in elastic half-space with void pores. Int. J. Numer. Anal. Methods Geomech. 17: 197-204

Ewing W M, Jardetzky W S, Press F 1957 Elastic waves in layered media (New York: McGraw Hill)

Nunziato J W, Cowin S C 1979 A non-linear theory of elastic materials with voids. Arch. Rational Mech. Anal. 72: 175-201

Pilant W L 1978 Elastic waves in the earth (New York: Elsevier Scientific)

Scalia A 1994 Shock waves in viscoelastic materials with voids. Wave Motion 19: 125-133

Yu T, Dresen L, Ruter H 1996 The influence of local irregularities on the propagation of Love waves. J. Appl. Geophys. 36: 53-65 